\title{
Postoperative Thrombotic Microangiopathy with the Combined Administration of Tranexamic Acid and Batroxobin: Two Case Reports
}

\author{
Min Hee Heo, Jung Hyeon Kim, Ji Yeon Kim \\ Department of Anesthesiology and Pain Medicine, Inje University Ilsan Paik Hospital, Inje University College of Medicine, Goyang, Korea
}

\begin{abstract}
Thrombotic microangiopathy (TMA) is a clinical syndrome characterized by microangiopathic hemolytic anemia, thrombocytopenia, and end-organ damage. When TMA is suspected, accurate diagnosis is required; however, differential diagnosis is often difficult because each patient has various clinical features, and the causes of triggers may overlap. We report two cases of TMA that developed after surgeries in patients, with the combined administration of tranexamic acid and batroxobin intraoperatively. We need to consider the risk of adverse effects of the concomitant use of tranexamic acid and batroxobin for anesthetic management.
\end{abstract}

Keywords: Batroxobin; Postoperative period; Thrombotic microangiopathies; Tranexamic acid

\section{INTRODUCTION}

Thrombotic microangiopathy (TMA) is a clinical syndrome characterized by microangiopathic hemolytic anemia (MAHA), thrombocytopenia, and end-organ damage [1]. TMA can be mainly classified into thrombocytopenic purpura (TTP) and hemolytic uremic syndrome (HUS). When TMA is suspected, accurate diagnosis is required, but a differential diagnosis is often difficult because each patient has varying clinical features, and the causes of triggers may overlap [2].

We report two cases of TMA that developed after surgeries in patients with the combined administration of tranexamic acid and batroxobin intraoperatively. We obtained informed consent from the patients for the publication of this case report.

\section{CASE REPORT}

\section{Case 1}

A 64-year-old woman $(156 \mathrm{~cm}, 72 \mathrm{~kg})$ diagnosed with hypertension and diabetes was scheduled for pylorus-preserving pancreaticoduodenectomy. At the operating room, her baseline blood pressure (BP) was 145/65 mm Hg, heart rate (HR) was $78 \mathrm{bpm}$, and oxygen saturation $\left(\mathrm{SpO}_{2}\right)$ was $97 \%$ on room air. Tranexamic acid $500 \mathrm{mg}$ and batroxobin $2 \mathrm{KU}$ (Klobusitzky unit) were administered intravenously before the skin incision.

The patient's preoperative and postoperative laboratory data are listed in Table 1. A peripheral blood smear (PBS) revealed schistocytes. Contrast-enhanced abdominal computed tomography on postoperative day 10 showed reduced parenchymal enhancement in both kidneys (Fig. 1A, B).

A disintegrin and metalloproteinase with thrombospondin motifs 13 protein (ADAMTS-13) activity was normal, and the polymerase chain reaction (PCR) results of the patient's stool samples were negative for Escherichia coli. Coombs tests were negative, and the complement component 4 (C4) level was normal, but the complement component 3 (C3) level was low to $72 \mathrm{mg} / \mathrm{dL}$. On postoperative day 1 , plasmapheresis and continuous renal replacement therapy (CRRT) were performed, which were followed by five sessions of plasmapheresis. On postoperative day 78, renal function recovered, and dialysis was stopped.

\section{Case 2}

A 58-year-old woman $(159 \mathrm{~cm}, 64 \mathrm{~kg})$ without any underlying disorders was scheduled for radical nephroureterectomy due to 
Table 1. Time series of patients' laboratory data

\begin{tabular}{|c|c|c|c|c|c|c|c|c|c|}
\hline \multirow{2}{*}{$\begin{array}{c}\text { Laboratory } \\
\text { variable }\end{array}$} & \multicolumn{4}{|c|}{ Case 1} & \multicolumn{4}{|c|}{ Case 2} & \multirow{2}{*}{ Normal range } \\
\hline & Preop 1 & OP day & POD 1 & POD 2 & Preop 1 & OP day & POD 2 & POD 4 & \\
\hline Diagnosis & \multicolumn{4}{|c|}{ Pancreatic head ductal adenocarcinoma } & \multicolumn{4}{|c|}{ Papillary urothelial carcinoma } & \\
\hline Operation & \multicolumn{4}{|c|}{ PPPD } & \multicolumn{4}{|c|}{ Radical nephroureterectomy } & \\
\hline Hemoglobin (g/dL) & 12.8 & 13.1 & 10.2 & 8.1 & 11.6 & 11.2 & 9.1 & 6.7 & $12.0-16.0$ \\
\hline Hematocrit (\%) & 39.4 & 38.8 & 29.7 & 23.7 & 34.8 & 32.8 & 27 & 19.3 & $34-48$ \\
\hline Platelet $\left(\times 10^{3}\right)$ & 284 & 185 & 38 & 14 & 197 & 151 & 62 & 39 & $150-400$ \\
\hline White blood cell $\left(\times 10^{3}\right)$ & 6.9 & 10 & 9.8 & 7.7 & 5.3 & 5.5 & 8.0 & 4.9 & 4-10 \\
\hline Creatinine $(\mathrm{mg} / \mathrm{dL})$ & 0.8 & 1.0 & 3.5 & 2.9 & 0.9 & 0.8 & 4.6 & 5.2 & $0.50-0.90$ \\
\hline Glomerular filtration rate $\left(\mathrm{mL} / \mathrm{min} / 1.73 \mathrm{~m}^{2}\right)$ & 76.6 & 58.5 & 13.3 & 16.6 & 67.8 & 71.6 & 9.9 & 8.5 & $>60$ \\
\hline Lactate dehydrogenase (U/L) & - & - & 3,100 & 1,575 & - & - & 645 & 608 & $135-214$ \\
\hline PT-INR & 1 & 1.26 & 1.2 & 1.13 & 1.0 & 1.1 & 1.1 & 1 & \\
\hline $\mathrm{aPTT}(\mathrm{sec})$ & 34.7 & 36.6 & 43.2 & 35.1 & 36.7 & 33.5 & 36 & 35.6 & $29-44$ \\
\hline Fibrinogen (mg/dL) & - & - & 268 & - & - & - & - & 261 & $180-400$ \\
\hline Complement component 3 (mg/dL) & - & - & 72 & - & - & - & 123 & - & 90-180 \\
\hline Complement component 4 (mg/dL) & - & - & 25 & - & - & - & 10 & 22 & $10-40$ \\
\hline Haptoglobin (mg/dL) & - & - & 15 & - & - & - & 22 & - & 30-200 \\
\hline
\end{tabular}

Preop, preoperative; OP, operation; POD, postoperative day; PPPD, pylorus-preserving pancreaticoduodenectomy; PT-INR, prothrombin time-international normalized ratio; aPTT, activated partial thromboplastin time.
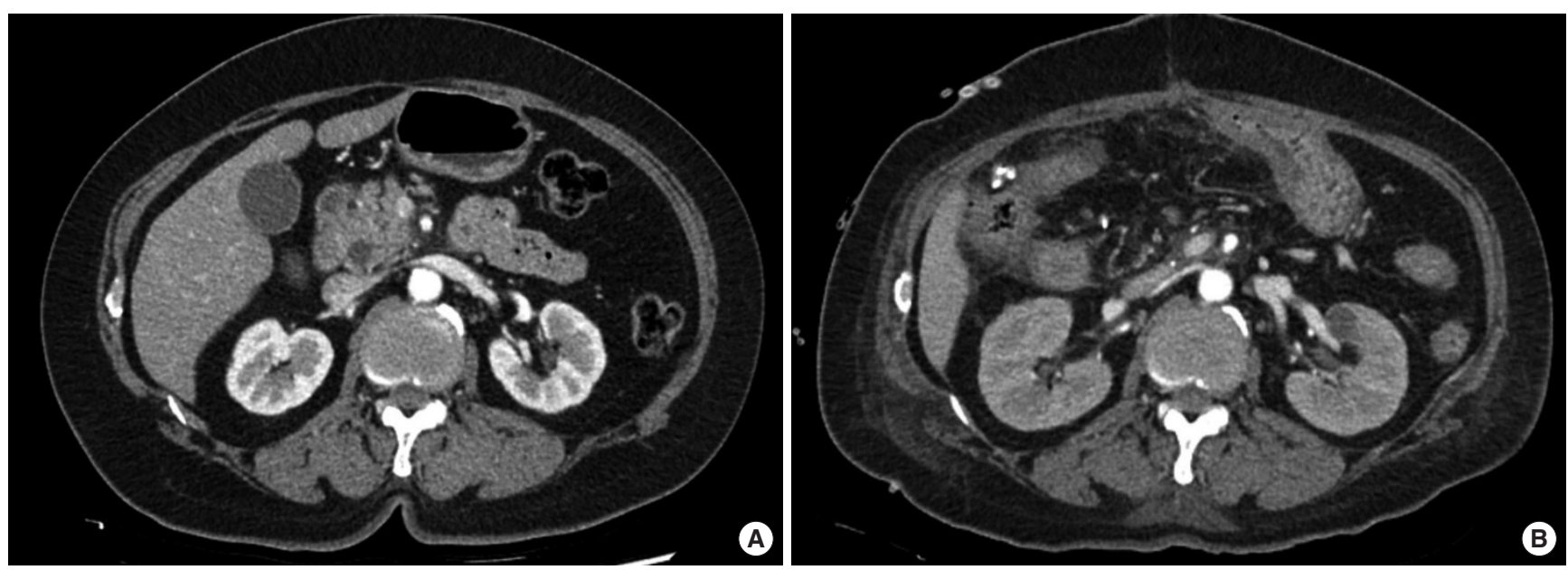

Fig. 1. Contrast-enhanced abdomen computed tomography shows normal kidney enhancement before the operation (A) and reduced parenchymal enhancement of both kidneys on postoperative day 10 (B).

renal pelvis cancer. At the operating room, her baseline $\mathrm{BP}$ was 173/94 mm Hg, $\mathrm{mR}$ was $71 \mathrm{bpm}$, and $\mathrm{SpO}_{2}$ was $100 \%$ on room air. During the operation, the mean BP was above $80 \mathrm{~mm} \mathrm{Hg}$. Tranexamic acid $500 \mathrm{mg}$ and batroxobin $2 \mathrm{KU}$ were administered before skin incision.

The patient's preoperative and postoperative laboratory data are listed in Table 1. Lactate dehydrogenase (LDH) was elevated to $645 \mathrm{U} / \mathrm{L}$, haptoglobin decreased to $22 \mathrm{mg} / \mathrm{dL}$, and PBS demonstrated schistocytes. ADAMTS-13 activity test, PCR of patient stool, and Coombs test were normal. The $\mathrm{C} 4$ level was normal, but the $\mathrm{C} 3$ level decreased to $75 \mathrm{mg} / \mathrm{dL}$.

From postoperative day 2, four sessions of plasmapheresis and six sessions of hemodialysis were performed. On postoperative day 20, renal function recovered, and dialysis was stopped, and the patient was discharged.

\section{DISCUSSION}

TMA is a rare disease that should be considered if a patient exhibits MAHA, thrombocytopenia, and ischemic end-organ dam- 
age [3]. MAHA is characterized by fragmented erythrocytes; laboratory findings of hemolysis, such as elevated serum $\mathrm{LDH}$ levels, decreased haptoglobin; and a negative Coombs test, which indicates the immune-mediated hemolysis of red blood cells [2].

In the cases described in this report, the patients had the typical triad of MAHA, thrombocytopenia, and acute kidney injury; these features were consistent with TMA. Once a diagnosis of TMA was established, it was essential to differentiate it from other causes of TMA. In order to rule out the TTP and Shiga toxin-producing E. coli (STEC)-HUS, an ADAMTS-13 activity test, and PCR were performed on the patient's stool samples, the results of which were negative. Therefore, TTP and STEC-HUS were ruled out. There was no evidence of coagulation factor deficiency, and the results of the Coombs test were negative. Therefore, disseminated intravascular coagulation and autoimmune disease were ruled out [4]. Besides, a low level of C3 and normal level of C4; suggest activation of the complement system and complement consumption; there is the possibility of atypical HUS (aHUS). The complement system's genetic testing may help an accurate diagnosis, but it was not conducted in these cases. But it is known that only about half of the patients who meet the clinical findings of aHUS have genetic abnormalities, aHUS cannot be excluded even if there is no genetic abnormality [5].

It is also necessary to rule out secondary TMA caused by malignant tumors, surgery, medications, transplantation, and malignant hypertension [4]. The patients described in this report had solid organ cancer, which had been surgically removed; there was no metastasis to other organs, and chemotherapy was not administered before or after surgery. Therefore, it was considered that the possibility of secondary TMA would be more likely due to the surgery, rather than cancer itself or the chemotherapeutic agents.

In the patients of this report, there is the possibility of the postsurgical TMA as the cause of secondary TMA. According to Sridharan et al. [6], the mechanism of postsurgical TMA involves an imbalance of von Willebrand factor (vWF) and ADAMTS13 due to endothelial damage, which is a feature of TTP, as well as the dysregulation of the complement pathway, which is a feature of aHUS. Indeed, cardiovascular surgery often causes a large amount of endothelial damage, resulting in an imbalance of vWF and ADAMTS13. By comparison, the underlying cause of TMA following less invasive surgery may be more directly attributed to the dysregulation of the complement pathway. As the patients in this report underwent relatively less invasive surgeries, the dysregulation of the complement pathway was considered to be the primary cause of postsurgical TMA. Nevertheless, it was difficult to clearly distinguish between aHUS and postsurgical TMA in two patients without genetic testing for aHUS in that they showed complement dysregulation in common.

In these cases, $500 \mathrm{mg}$ of tranexamic acid and $2 \mathrm{KU}$ of batroxobin were administered simultaneously to reduce intraoperative bleeding. Nagabhushan et al. [7] reported that the intraoperative administration of a combination of batroxobin (1 KU intravenously 15 minutes before skin incision, followed by $0.5 \mathrm{KU}$ intramuscularly) and tranexamic acid (10 mg/kg bolus intravenously 15 minutes before skin incision, followed by $1 \mathrm{mg} / \mathrm{kg} / \mathrm{hr}$ ) significantly reduced blood loss during spinal surgery, without increasing the risk of thrombosis. These two agents are antifibrinolytic and induce clot formation, and seem to have a synergistic effect [8]. Previous studies have reported, rare adverse effects, such as renal cortical necrosis, with the daily administration of tranexamic acid ( $3 \mathrm{~g}$ ) over a period of 4 to 5 days $[9,10]$. However, in our report, $500 \mathrm{mg}$ of tranexamic acid and $2 \mathrm{KU}$ of batroxobin were used, resulting in thrombotic complications. Furthermore, none of the underlying diseases and past history related to thrombotic events were present in the two patients in this case.

Given the potential dysregulation of the complement system among patients in this report, it was considered that microthrombi formation might have been a consequence of the synergistic effects of the concomitant tranexamic acid and batroxobin administration. Although it was not clear whether the combined use of the hemostatic agents directly affected the triggering of TMA, they may have had a role in its exacerbation.

In conclusion, for the anesthetic management of patients with a risk of thrombosis, such as those undergoing cancer surgery, we need to consider the risk of adverse effects associated with the concomitant use of tranexamic acid and batroxobin.

\section{CONFLICT OF INTEREST}

No potential conflict of interest relevant to this article was reported.

\section{REFERENCES}

1. Sridharan M, Go RS, Willrich MA. Atypical hemolytic uremic syndrome: review of clinical presentation, diagnosis and management. J Immunol Methods 2018;461:15-22. 
2. Moake JL. Thrombotic microangiopathies. N Engl J Med 2002;347:589600 .

3. George JN, Nester CM. Syndromes of thrombotic microangiopathy. N Engl J Med 2014;371:654-66.

4. Cheong HI, Jo SK, Yoon SS, Cho H, Kim JS, Kim YO, et al. Clinical practice guidelines for the management of atypical hemolytic uremic syndrome in Korea. J Korean Med Sci 2016;31:1516-28.

5. Yasuda S, Yamamoto M, Fukuda T, Ohtsuka Y, Miura O. Postoperative atypical hemolytic uremic syndrome treated successfully with eculizumab. Intern Med 2016;55:1171-5.

6. Sridharan M, Hook CC, Leung N, Winters JL, Go RS; Mayo Clinic Complement Alternative Pathway-Thrombotic Microangiopathy DiseaseOriented Group. Postsurgical thrombotic microangiopathy: case series and review of the literature. Eur J Haematol 2019;103:307-18.
7. Nagabhushan RM, Shetty AP, Dumpa SR, Subramanian B, Kanna RM, Shanmuganathan R. Effectiveness and safety of batroxobin, tranexamic acid and a combination in reduction of blood loss in lumbar spinal fusion surgery. Spine (Phila Pa 1976) 2018;43:E267-73.

8. $\mathrm{Xu} \mathrm{C}, \mathrm{Wu} \mathrm{A}$, Yue Y. Which is more effective in adolescent idiopathic scoliosis surgery: batroxobin, tranexamic acid or a combination? Arch Orthop Trauma Surg 2012;132:25-31.

9. Koo JR, Lee YK, Kim YS, Cho WY, Kim HK, Won NH. Acute renal cortical necrosis caused by an antifibrinolytic drug (tranexamic acid). Nephrol Dial Transplant 1999;14:750-2.

10. Odabas AR, Cetinkaya R, Selcuk Y, Kaya H, Coskun U. Tranexamic-acid-induced acute renal cortical necrosis in a patient with haemophilia A. Nephrol Dial Transplant 2001;16:189-90. 